\title{
The predictive power of spallation models for isotopic cross sections
}

\author{
Udai Singh $^{1}$, Detlef Filges ${ }^{2}$, Frank Goldenbaum ${ }^{2}$, Bogusław Kamys ${ }^{1, a}$, Zbigniew Rudy ${ }^{1}$, and Sushil K. Sharma ${ }^{1}$ \\ 1 The Marian Smoluchowski Institute of Physics, Jagiellonian University, Łojasiewicza 11, 30-348 Kraków, Poland \\ 2 Institut fuer Kernphysik, Forschungszentrum Juelich, 52425 Juelich, Germany
}

Received: 23 March 2018 / Revised: 28 May 2018

Published online: 26 June 2018

(C) The Author(s) 2018. This article is published with open access at Springerlink.com Communicated by F. Gulminelli

\begin{abstract}
The experimental cross sections of isotopically identified products of spallation reactions induced by ${ }^{136} \mathrm{Xe}$ projectiles at $1 \mathrm{GeV} /$ nucleon on hydrogen target were compared with predictions of a two-step model. The first stage of the reaction was described by the INCL++ model (version 5.3) of an intranuclear cascade of nucleon-nucleon and pion-nucleon collisions whereas the second stage was analyzed by means of four different models; ABLA07, GEM2, GEMINI ++ and SMM. Due to the fact that the experimental data cover a very broad range of elements; from $\mathrm{Li}(Z=3)$ to $\mathrm{Ba}(Z=56)$, the analysis could impose severe constraints on the applied reaction models. The quality of data reproduction by the theoretical models is discussed. Some systematic deviations of the theoretical predictions from the experimental results are observed.
\end{abstract}

\section{Introduction}

Spallation of atomic nuclei due to their collisions with protons plays an important role in many physical phenomena as, e.g., in evolution of heavy stars, in modification of the nuclear content of cosmic rays during their propagation through the interstellar space [1]. It is also crucial in many technical applications as, e.g., accelerator-driven subcritical reactors as well as in radioactive waste transmutation [2], construction and application of spallation neutron sources [3]. Thus, the knowledge of spallation cross sections is indispensable for the understanding of these physical phenomena and for many technical applications. Since some of the important targets and/or product nuclei are unstable the determination of the appropriate spallation cross sections cannot be accomplished by straightforward measurements but it has to be obtained from reliable theoretical models.

The predictive power of the models must be tested by comparison of theoretical cross sections to experimental data obtained for different nuclei interacting with protons at various energies. In the last years a set of systematic experimental studies was performed using the inverse kinematics, i.e. bombarding the hydrogen target by the accelerated atomic nuclei; Fe [4-6], Au [7-9], $\mathrm{Pb}[10,11]$, $\mathrm{U}[12-14]$, etc. which allowed to extract cross sections for isotopically identified products in a broad range of their atomic and mass numbers.

\footnotetext{
a e-mail: ufkamys@cyf-kr.edu.pl (corresponding author)
}

In the present work a task is undertaken to check the ability of several theoretical models which are frequently used in the literature to reproduce the isotopic cross sections $\sigma(Z, N)$ measured for ${ }^{136} \mathrm{Xe}+p$ system at energy of $1 \mathrm{GeV} /$ nucleon [15]. The data under investigation cover a very large range of the produced elements, i.e., from $\mathrm{Li}$ $(Z=3)$ to $\mathrm{Ba}(Z=56)$. This work is a continuation of the recent study [16] in which the same reactions have been analyzed at lower energy $(0.5 \mathrm{GeV} /$ nucleon $)$. Then the investigated data [17] contained only heavy reaction products, i.e., nuclei from $\mathrm{Nb}(Z=41)$ to $\mathrm{Ba}(Z=56)$. It was found that all applied models gave reasonable qualitative agreement with the data whereas a perfect, quantitative agreement was not achieved. Due to the fact, that the present data cover a much broader range of the reaction products it may be conjectured that they will offer much stronger demands for the reaction models than the previously analyzed isotopic cross sections.

The model calculations of the isotopic cross sections are discussed in the second section of the paper. The experimental and model cross sections are presented in figures as function of the mass number $\mathrm{A}$ for all produced elements. This enabled us to qualitatively compare the model cross sections with the data. In the next section the $A$-deviation factor [16] is applied to investigate quantitatively the agreement of the model and experimental cross sections. The goal of this analysis is to judge on the validation of the models and to establish their ranking. Results are summarized and discussed in the last section of the paper. 


\section{Analysis of isotopic cross sections $\sigma(\mathbf{A} \mid \mathbf{Z})$}

The present analysis was performed in the frame of the two-stage microscopic model. The first step of the process was described as intranuclear cascade of nucleon-nucleon and pion-nucleon collisions leading to the equilibrated, excited remnant nucleus. It was realized by the INCL++ (version 5.3) model [18]. The de-excitation of the equilibrated heavy products of the first step of the process has been calculated in the frame of four different models: ABLA07 [19], GEM2 [20,21], GEMINI++ [22,23] and SMM [24-27]. The physical assumptions of the models as well as the details of their realization may be found in the references cited above. Here, only the most important information is summarized.

The INCL $++[18]$ is the $\mathrm{C}++$ version of the INCL4. 6 FORTRAN77 code [28]. It is physically almost identical with INCL4.6 when applied to proton induced reactions. The main difference consists in different treatment of the pion potential, using another prescription for the nucleon-nucleon "interaction range", and application of modified preparation of the light target nuclei $(A<4)$. A very important extension of the INCL++ in comparison to INCL4. 6 is the possibility to analyze collisions of light ions $(A \leq 18)$ with nuclei. For our purposes, i.e. the description of proton induced reactions on ${ }^{136} \mathrm{Xe} \mathrm{nu}-$ clei the INCL ++ may be treated as physically equivalent to INCL4.6. The model describes the emission of nucleons and pions from the cascade of nucleon-nucleon and nucleon-pion collisions but allows also for the emission of complex particles $(Z<5, A<9)$. The last process is treated as a surface coalescence of the outgoing nucleon with the nucleons which appear to be closely to it in the momentum and coordinate space. It should be noted that the cross sections for $A=8$ are always overestimated by the model since it assumes that all heavier groups of nucleons created due to the coalescence - even if the individually small- give a contribution to the emission of $A=8$ particles.

The models of the second stage of the reaction assume that the excited remnant of the cascade achieves the thermodynamical equilibrium. All of them allow for evaporation of nucleons and complex particles but differ in the assumptions concerning the realization of this process. The GEM2 [20,21] uses the formalism of Weisskopf and Ewing [29] in which probability of emission of particles is determined by the cross sections for reaction reversed in time as well as by the density of states of the parent and daughter nuclei. The GEM2 takes into consideration evaporation of complex particles up to magnesium $(Z=12)$. The model enables one to calculate also the fission of excited nuclei according to the prescription due to Atchison [30], however, the fission is taken into consideration only for nuclei with atomic number $Z>60$. Therefore, in the present reactions for the $p+{ }_{54} \mathrm{Xe}$ nuclear system this mode of the de-excitation is switched off.

In the ABLA07 statistical model the evaporation of particles is also calculated according to the WeisskopfEwing formalism but the influence of the angular momentum is additionally introduced albeit in some simplified manner. The change of the angular momentum is sampled according to a Gaussian distribution with specifically chosen parameters. The emission of any stable nucleus up to half the mass of the compound nucleus is possible. The fission and multifragmentation is also taken into consideration in the ABLA07 model. The last process is allowed for nuclei with the temperature which exceeds the following mass-dependent threshold (in $\mathrm{MeV}$ ):

$$
T_{\text {freeze-out }}=\max [5.5,9.33 \exp (-0.00282 \times A)] .
$$

The excited nucleus breaks up into fragments whose mass follows an empirical power-law distribution. The excited fragments may evaporate particles which is also calculated by the Weisskopf-Ewing model. The fission process is allowed to compete with evaporation and multifragmentation. It is treated as a diffusion process described by the Fokker-Planck equation. The solution of the equation is analytically parametrized, producing the time dependent fission width. Details of the model may be found in ref. [19].

The Statistical Multifragmentation Model SMM of Botvina et al. [24-26] is described in detail in ref. [27]. The model assumes that the thermalized remnant of the first step of the proton-nucleus collision expands to a certain volume and then breaks up into nucleons and hot fragments. The break-up appears at a density of one-sixth to one-third of the normal nuclear density. The probability $w_{j}$ of the given break-up channel $j$ is determined by the excitation energy $E^{*}$ dependent entropy $S_{j}\left(E^{*}\right)$

$$
w_{j} \sim \exp S_{j}\left(E^{*}\right)
$$

The model treats the compound nucleus as one of the decay channels therefore the transition from evaporation of particles from the compound nucleus to its multifragmentation is decided on the basis of the available phase space. The fragments of the decaying nucleus propagate independently in their mutual Coulomb fields taken into account via the Wigner-Seitz approximation and undergo secondary decays. The deexcitation of fragments with mass number larger than 16 is treated by the evaporation according to the Weisskopf-Ewing formalism [29] (up to ${ }^{18} \mathrm{O}$ ) or by the fission model in formalism of BohrWheeler [33]. Decay of fragments with the mass number A smaller than 16 is described by the Fermi break-up model [27].

The GEMINI++ describes the decay of a compound nucleus by a series of binary divisions until the resulting products are unable to undergo further decay $[22,23]$. The GEMINI++ code uses the Hauser-Feshbach formalism [31] of evaporation of light particles (in the default version with $Z$ up to 3 ) which explicitly treats and conserves angular momentum. The partial decay widths for more symmetric divisions of the compound nucleus are calculated according to Moretto generalized transitionstate formalism [32] which describes well the decay of light compound nuclei. For heavier nuclei the Bohr-Wheeler formalism [33] is used for symmetric fission, and the width of the mass distributions of the fission fragments is interpolated from systematics. However, the Moretto formalism is kept for asymmetric divisions of heavy systems. 
The calculations of the isotopic production cross sections measured by Napolitani et al. [15] for the system ${ }^{136} \mathrm{Xe}+$ hydrogen at $1 \mathrm{GeV}$ per nucleon were performed with default values of the parameters of all the models. Therefore it was possible to judge the predictive power of the applied models.

Results of the calculations are presented in figs. 1, 2 and 3 for light $(2<Z<15)$, intermediate mass $(14<$ $Z<33)$ and heavy $(32<Z<57)$ reaction products, respectively.

The qualitative agreement of the theoretical cross sections with the data will be discussed below for each range of the products separately. It is obvious from the inspection of fig. 1 that the GEM2 model underestimates systematically all the data. Furthermore, the theoretical cross sections decrease much faster with the increasing of the atomic number $Z$ than the data. In result the theoretical cross sections of GEM2 are negligibly small for $Z>9$. The isotopic cross sections predicted by other models do not deviate so significantly from the data, especially there is not visible such a systematic underestimation of the data as well as its increase with the atomic number. All the models produce bell shaped mass distributions of the isotopic cross sections similar to the distributions of the experimental cross sections. However, none of the models reproduces exactly the behaviour of the data. Absolute values of the cross sections predicted by ABLA07 for $Z<9$ are smaller than those of the data (with exception of the carbon and oxygen isotopes which seem to be well reproduced). The shape of the mass distributions is reasonably well reproduced for given element with exception of fluorine where the distribution is shifted towards small masses and aluminium as well as silicon where the theoretical distributions are too broad. The SMM model systematically under-predicts the cross sections for heaviest isotopes whereas it overestimates the cross sections for lightest isotopes (with exception of nitrogen where all the models do not work well). GEMINI++ seems to produce proper width and position of the cross section distributions, however, it usually under-predicts values of the cross sections.

The situation is different for elements with $14<Z<$ 33. The experimental and theoretical mass distributions of the isotopic cross sections are presented for these elements in fig. 2. As can be seen the GEM2 model does not produce any elements with $Z<30(\mathrm{Zn})$. Starting from Zn the theoretical cross sections evaluated with GEM2 appear to be non-negligible but they are more than one order of magnitude smaller than the experimental ones. Thus, the GEM2 cross sections for the discussed range of elements are completely unrealistic. Other models predict the cross sections which are of the same order of magnitude as the data and, furthermore, the shape of the mass distributions of the theoretical cross sections is similar to the shape of experimental distributions. However, systematic deviations of the theoretical cross sections from the data are observed. The mass distributions produced by ABLA07 are too broad in the comparison to the experimental ones what always leads to the overestimation of the isotopic cross sections for all isotopes with mass larger than the most populated isotope and frequently also for isotopes with the smallest masses. The opposite situation is observed for the cross sections predicted by the SMM model. In this case the cross sections for isotopes with the smallest mass are systematically overestimated whereas those for the largest masses are systematically underestimated. Thus, in spite of the similarity of the shape of the mass dependence produced by the SMM model and that observed in the experiment the absolute values of the cross sections are systematically overestimated or underestimated by the model. Position of the maximum of the mass distribution of the isotopic cross sections as well as its width is in most cases well reproduced by GEMINI++, however, this model systematically under-predicts the absolute values of the cross sections what is most pronounced in the neighbourhood of the maxima of the distributions.

The following qualitative conclusions may be derived from inspection of fig. 3 which presents the isotopic cross sections measured and calculated for the elements with $32<Z<57$. The magnitude of the cross sections predicted by GEM2 model increases with increase of the atomic number of the products. At $Z=33$ the model cross sections are an order of magnitude smaller than the experimental ones but starting from $Z=40$ they agree quite well with the data. The shape of the mass distribution of the isotopic cross sections predicted by GEM2 for $Z>40$ becomes quite similar to that of the experimental one which is smooth and almost symmetrical up to $Z=47$ (Ac). The other models, i.e., ABLA07, SMM and GEM$\mathrm{INI}++$ reproduce well the shape of the mass dependence and position of its maximum for all elements starting from $Z=33$ (As) up to $Z=46(\mathrm{Pd})$. However, the width of the mass of theoretical and experimental distributions agrees only for ABLA07 and GEMINI++. The SMM model usually produces too narrow distributions. The experimental mass distributions for the $Z=47$ to $Z=52$ range of elements (i.e., Ac-Te) become asymmetric with the larger values of the cross sections for the isotopes with large mass number. This behaviour is only partly reproduced by the theoretical models. All of them predict too flat distributions in comparison to the experimental ones. As a consequence the values of the experimental cross sections agree well with the model cross sections for the lightest isotopes of a given element, they are slightly underestimated for isotopes with average masses whereas they are significantly overestimated (even two orders of magnitude) for the heaviest isotopes. The situation changes for reaction products with the largest atomic numbers, i.e., for $Z=53$ and $Z=54$ (I and Xe). Then the distribution of the isotopic cross sections monotonically increases with the mass of isotopes. All the models reproduce this change of the character of the distributions as well as the magnitude of the cross sections. The GEM2 model seems to be the poorest in reproduction of $\mathrm{Xe}(Z=54)$ isotopic cross sections, however it describes well the isotopic cross sections for I $(Z=53)$ and $\operatorname{Cs}(Z=55)$. The experimental distribution of the isotopic cross sections for the element with the largest $Z(Z=56)$, i.e., for $\mathrm{Ba}$, is systematically overestimated by all theoretical models. 

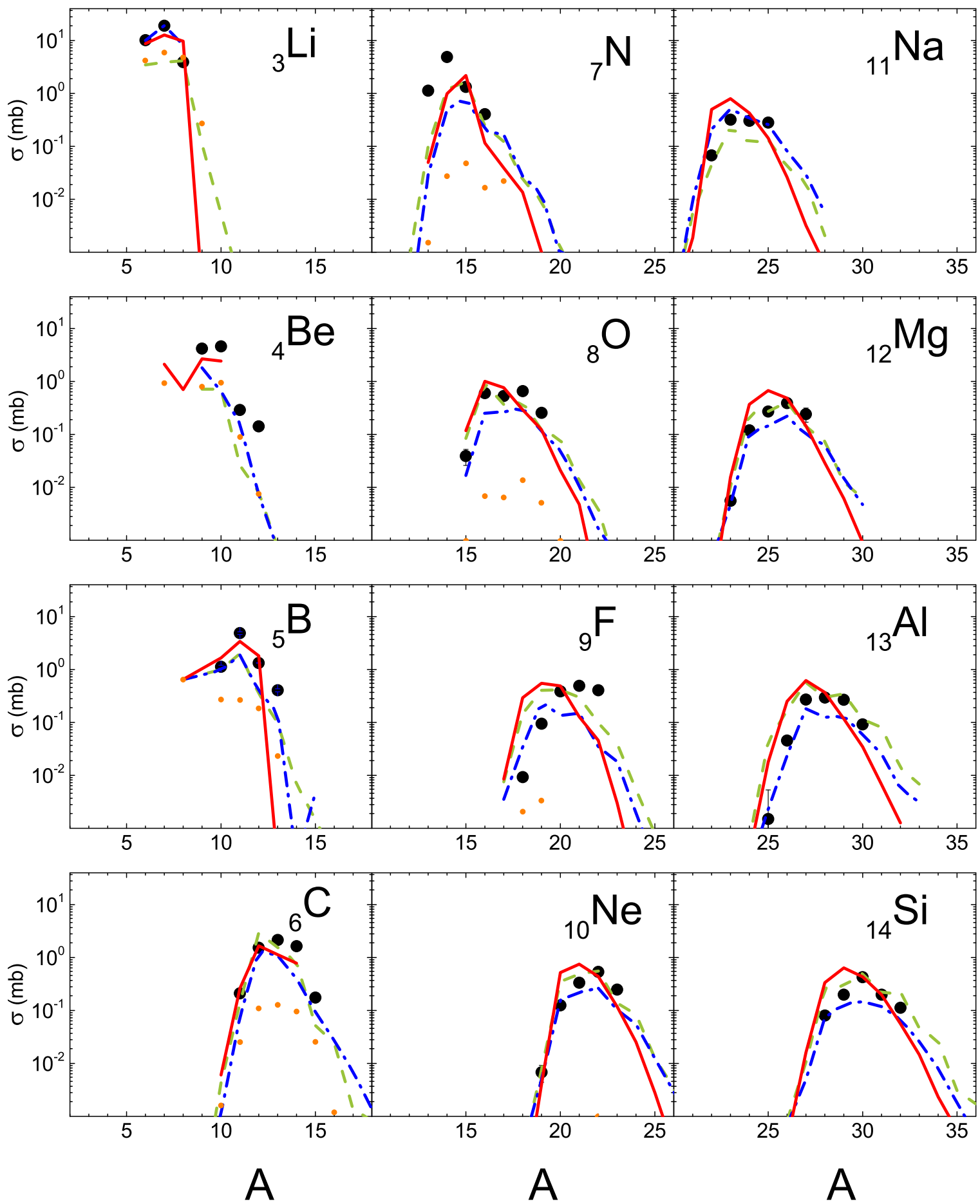

Fig. 1. Isotopic cross sections of IMFs with $Z=3-14$ from $\mathrm{p}+{ }^{136} \mathrm{Xe}$ collisions at energy $\mathrm{T}\left({ }^{136} \mathrm{Xe}\right)=1000 \mathrm{AMeV}[15](\mathrm{black}$ dots) together with predictions of the INCL++ (version 5.3) model for the first stage of the reaction coupled to four models; ABLA07 (green dashed line), GEM2 (orange dots), GEMINI++ (blue dash-dotted line) and SMM (red solid line) used for the second step of the reaction. Note the absence of the theoretical points due to the GEM2 for elements with $Z>9$. 

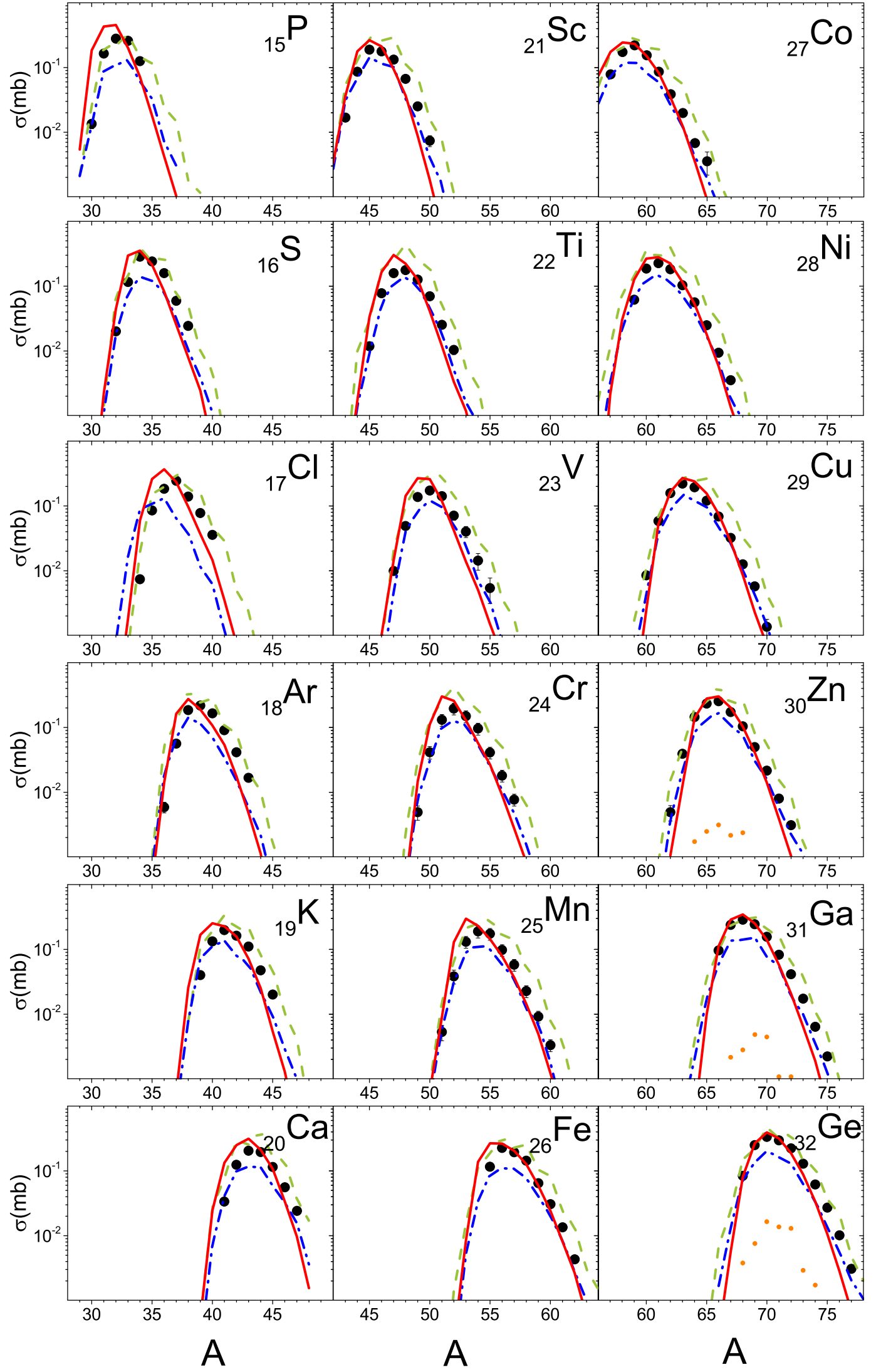

Fig. 2. Same as fig. 1, but for $Z=15-32$. Note the absence of the theoretical points due to the GEM2 for elements with $Z<30$. 

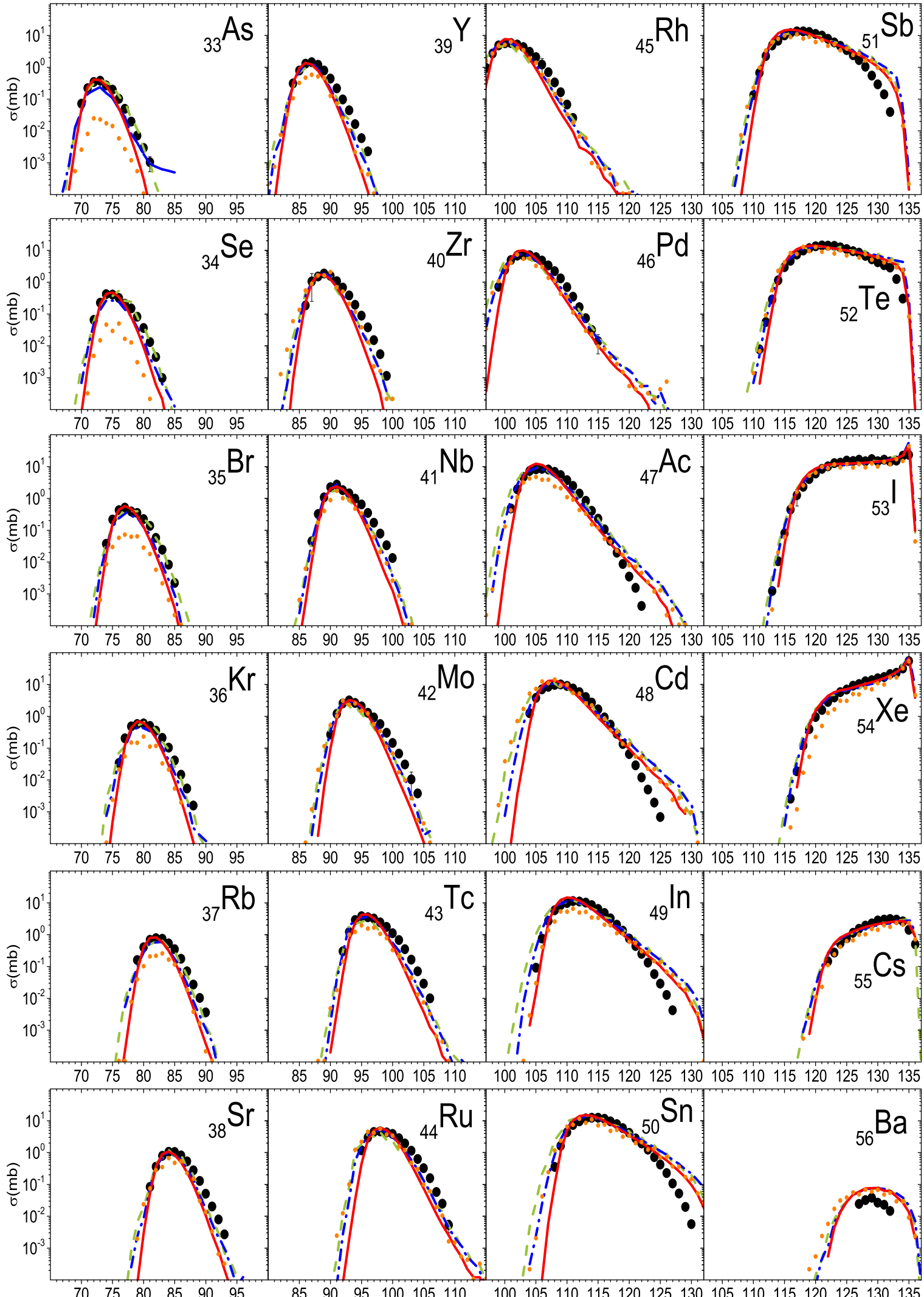
A
A
A
A

Fig. 3. Same as fig. 1, but for $Z=33-56$. 

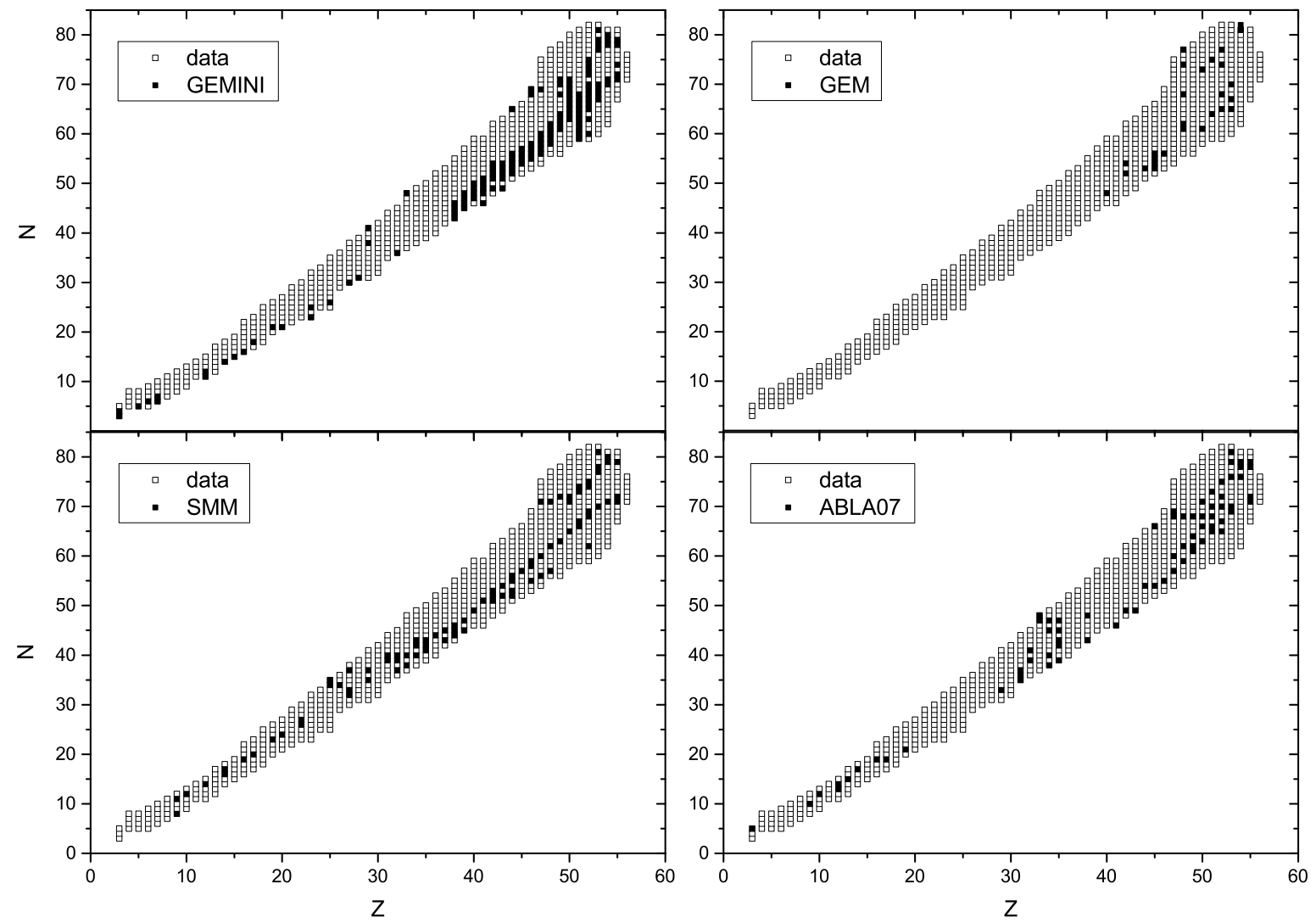

Fig. 4. The values $(Z, N)$ of experimentally obtained isotopic cross section $[15]$ for ${ }^{136} \mathrm{Xe}+\mathrm{p}$ at energy $1 \mathrm{GeV} / \mathrm{nucleon}$ (empty squares) and the values $(Z, N)$ at which the relative deviation between calculated and experimental cross sections $2 \mid \sigma^{\text {cal }}-$ $\sigma^{e x p} \mid /\left(\sigma^{c a l}+\sigma^{e x p}\right)$ is smaller than $10 \%$ (full squares). The left upper panel contains results of GEMINI++, the right upper contains results of GEM2, the left lower panel contains results of SMM and the right lower panel contains those of ABLA07.

The detailed discussion of the agreement between model and experimental isotopic cross sections presented above does not allow to make a simple, general overview of the quality of description of all the data by all models. To allow for such an overview the following procedure was applied.

All isotopes for which the production cross sections were determined in the experiment [15] are presented as empty squares in the two dimensional plot $(Z-N)$ in fig. 4. The isotopes for which the model cross sections do not deviate more than $10 \%$ from the data are shown as full squares. This specific value of the relative deviation was chosen somewhat arbitrary taking into consideration that the typical relative errors estimated for the most abundant isotopes in the experiment [15] are equal to $5 \%-6 \%$ and do not overcome $20 \%$. It may be concluded after inspection of fig. 4 that such a representation allows to observe characteristic behaviour of the quality of reproduction of the data by different models:

i) The number of well described data is rather small; about $12 \%$ of the experimental cross sections are well reproduced by ABLA07, SMM and GEMINI++ whereas only about $4 \%$ in the case of the GEM2 model.

ii) The cross sections for products with large atomic number $Z$ are more frequently reproduced by the models than those for products with small $Z$. This is especially pronounced in the case of GEM2 where the only reproduced experimental cross sections are those for large $Z$.

iii) In the case of GEMINI++ several neighbouring isotopes of the same element with the large $Z$ are very well reproduced what is not the case for other models. This indicates that for these elements GEMINI++ well reproduces the shape of the $N$-dependence of the experimental cross sections (at least for the largest $N$ values, cf. fig. 3). A quite different situation is present for SMM where two or three lines of well reproduced $(Z-N)$ cross sections are visible. It is caused by the fact that the shape of the mass dependence of isotopic cross sections predicted by SMM is different than the experimental shape. Due to this the experimental and theoretical $N$ distributions for given $Z$ are crossing at two or three $N$ values, cf. fig. 3 .

iv) The data for elements with $30<Z<40$ are not reproduced by GEMINI++ and GEM2 but the ABLA07 and SMM predictions agree well with the data.

v) The data for elements with $20<Z<30$ are not described by GEM2 and ABLA07 whereas SMM and GEMINI++ work well for this range of the atomic number.

vi) Isotopic cross sections for elements with $10<Z<$ 20 are not reproduced by GEM2 but some of them are well described by other models.

It is worth emphasizing that different models describe well different isotopes for this range of atomic number: 


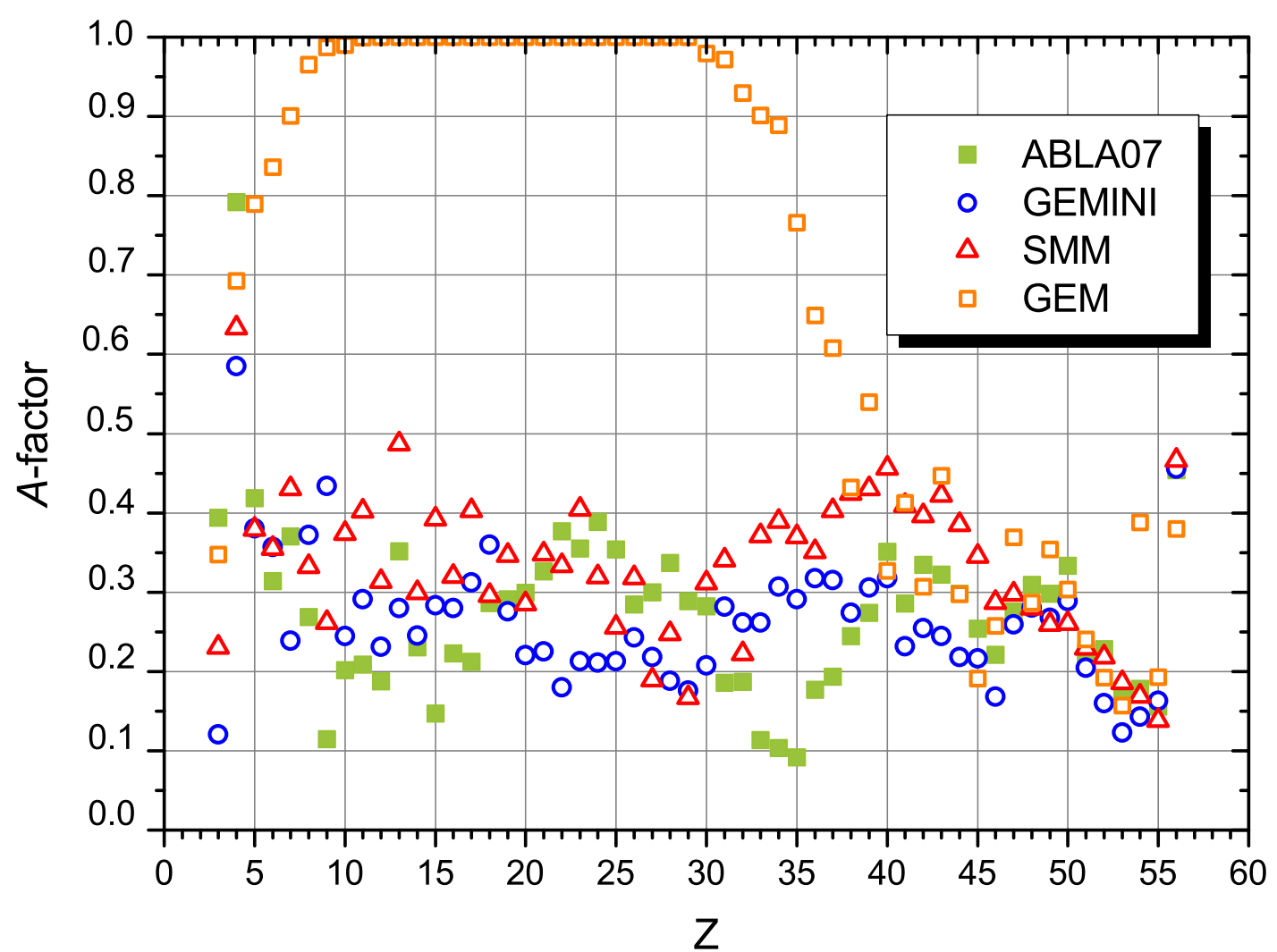

Fig. 5. Values of the $A$ deviation factor evaluated according to eq. (1). Green, solid squares represent results obtained with ABLA07, orange, open squares correspond to GEM2, red, open triangles to SMM whereas blue, open circles show values evaluated with GEMINI++.

GEMINI++ is good for the smallest $N$-values whereas ABLA07 and SMM for average $N$, i.e., ABLA07 and SMM reproduce well the maximal isotopic cross sections for given $Z$. This specific behaviour is caused by the shift of the mass distributions produced by ABLA07 and SMM towards small $N$ values with respect to the experimental distributions, cf. fig. 1.

\section{The A-deviation factor}

The present discussion of the quality of the agreement between model and experimental cross sections concerned the individual isotopic cross sections. Frequently the question arises of the agreement of the theoretical cross sections with the data averaged over all isotopes of a given element. It was shown in the previous paper [16], which deals with the same reactions in the ${ }^{136} \mathrm{Xe}+\mathrm{p}$ system but at twice smaller energy, that a specific deviation factor may serve well for determination of the quality of the agreement between model and experimental cross sections. This so called $A$-factor is defined as follows:

$$
A \equiv \frac{1}{N} \sum_{i=1}^{N} \frac{\left|\sigma_{i}^{\exp }-\sigma_{i}^{c a l}\right|}{\sigma_{i}^{\exp }+\sigma_{i}^{c a l}}
$$

where the sum runs over all isotopes of a given produced element. Values of this factor belong to the interval $[0,1]$ being equal to zero for the perfect agreement between theoretical and experimental cross sections and increasing monotonically with the deterioration of the agreement. It is important to note that small values of this factor, which correspond to $\sigma_{i}^{\exp } \approx \sigma_{i}^{c a l}$, may be interpreted as a half of the average relative distance between the compared cross sections.

The $A$-factor values calculated for all the data and for corresponding combinations of models are presented in fig. 5. As can be seen in this figure the $A$-factor evaluated with the GEM2 model cross sections differs strongly from all others. It has values close or equal to one for all elements with $Z$ between 9 and 30 . This is due to the fact that GEM2 does not produce ejectiles with such values of the atomic number $Z$. Other models, which better reproduce the data have smaller $A$-factor values varying between 0.1 and 0.6 .

The following interesting conclusions may be derived from inspection of fig. 5:

i) The data with $47<Z<55$ are equally well reproduced by all the models. This is the range of elements which are mainly produced by the evaporation of nucleons from the excited remnant of the intranuclear cascade.

ii) For products with $40<Z<47$ GEMINI++ is the best in reproducing the data, SMM is the worst whereas 
results of ABLA07 are randomly better or poorer than those of GEM2.

iii) The ABLA07 gives distinctly the best description of the products with $30<Z<40$. GEMINI++, SMM and GEM2 lead to the poorer reproduction of the data with the quality decreasing in the indicated sequence.

iv) The situation changes for products with $18<Z<30$ where the GEMINI++ offers the best description of the data. ABLA07 and SMM compete to provide second the best reproduction of the experimental cross sections. The GEM2, which does not produce ejectiles for this range of atomic number is completely not applicable.

v) The lowest range of atomic numbers, i.e., $Z<19$ seems to be the best described by ABLA07 whereas GEMINI++ and SMM give comparable, slightly poorer description.

To summarize this analysis quantitatively, the ranks of the models based on values of the $A$-factor were collected in table 1 for all elements produced in the studied reactions. A smaller rank of the model for a given element means that the model leads to a smaller $A$-factor value and therefore corresponds to a better description of the data. If two models give practically the same value of the $A$ factor then the arithmetic average of their ranks is quoted in table 1 for both models. The sum of the ranks of a given model performed over all observed elements can be treated as a quantitative measure of the quality of the description of all the data by the model under consideration.

As can be seen in table 1 the quality of the general description of the data is the best for GEMINI++ (sum of the ranks is equal to 91 ), the second place in the description is granted to ABLA07 (sum equal to 116), the third is due to SMM (sum equal to 138) and the poorest description is obtained with GEM2 (sum of ranks equal to 195$)$.

\section{Summary}

A very rich set of production cross sections (over 600 cross sections) measured by Napolitani et al. [15] with isotopic identification of the products for ${ }^{136} \mathrm{Xe}+\mathrm{p}$ collisions at $1 \mathrm{GeV}$ per nucleon was compared with predictions of a two-step microscopic model. The first stage of the reaction was analyzed in the frame of the INCL++ model [18] which treats the proton-nucleus collision as a sequence of nucleon-nucleon and nucleon-pion collisions leaving the equilibrated, excited remnant nucleus. The second stage of the reaction was described by four different models: ABLA07 [19], GEM2 [20,21], GEMINI++ [22,23] and SMM [24-27] which assume different scenarios of the de-excitation of the remnant of the intranuclear cascade. All calculations were performed using the default values of the applied theoretical models with the aim to study the predictive power of the models in respect to the determination of isotopic cross sections.

It was found that ABLA07, SMM and GEMINI++ reproduce the main properties of the $Z$ and $A$ dependence of
Table 1. Ranks of various model predictions for isotopic distributions of residua from $\mathrm{Xe}+\mathrm{p}$ collisions at $1 \mathrm{GeV} /$ nucleon [15] according to values of the $A$-deviation factor.

\begin{tabular}{|c|c|c|c|c|}
\hline \multirow[b]{2}{*}{ Ejectile } & \multicolumn{4}{|c|}{ The ranks of the models } \\
\hline & ABLA07 & GEM2 & GEMINI++ & SMM \\
\hline${ }_{3} \mathrm{Li}$ & 4 & 3 & 1 & 2 \\
\hline${ }_{4} \mathrm{Be}$ & 4 & 3 & 1 & 2 \\
\hline${ }_{5} \mathrm{~B}$ & 3 & 4 & 2 & 1 \\
\hline${ }_{6} \mathrm{C}$ & 1 & 4 & 2 & 3 \\
\hline${ }_{7} \mathrm{~N}$ & 2 & 4 & 1 & 3 \\
\hline${ }_{8} \mathrm{O}$ & 1 & 4 & 3 & 2 \\
\hline${ }_{9} \mathrm{~F}$ & 1 & 4 & 3 & 2 \\
\hline${ }_{10} \mathrm{Ne}$ & 1 & 4 & 2 & 3 \\
\hline${ }_{11} \mathrm{Na}$ & 1 & 4 & 2 & 3 \\
\hline${ }_{12} \mathrm{Mg}$ & 1 & 4 & 2 & 3 \\
\hline${ }_{13} \mathrm{Al}$ & 2 & 4 & 1 & 3 \\
\hline${ }_{14} \mathrm{Si}$ & 1 & 4 & 2 & 3 \\
\hline${ }_{15} \mathrm{P}$ & 1 & 4 & 2 & 3 \\
\hline${ }_{16} \mathrm{~S}$ & 1 & 4 & 2 & 3 \\
\hline${ }_{17} \mathrm{Cl}$ & 1 & 4 & 2 & 3 \\
\hline${ }_{18} \mathrm{Ar}$ & 1 & 4 & 3 & 2 \\
\hline${ }_{19} \mathrm{~K}$ & 2 & 4 & 1 & 3 \\
\hline${ }_{20} \mathrm{Ca}$ & 3 & 4 & 1 & 2 \\
\hline${ }_{21} \mathrm{Sc}$ & 3 & 4 & 1 & 2 \\
\hline${ }_{22} \mathrm{Ti}$ & 3 & 4 & 1 & 2 \\
\hline $23 \mathrm{~V}$ & 2 & 4 & 1 & 3 \\
\hline${ }_{24} \mathrm{Cr}$ & 3 & 4 & 1 & 2 \\
\hline${ }_{25} \mathrm{Mn}$ & 3 & 4 & 1 & 2 \\
\hline${ }_{26} \mathrm{Fe}$ & 3 & 4 & 2 & 1 \\
\hline${ }_{27} \mathrm{Co}$ & 3 & 4 & 2 & 1 \\
\hline $28 \mathrm{Ni}$ & 3 & 4 & 1 & 2 \\
\hline${ }_{29} \mathrm{Cu}$ & 3 & 4 & 2 & 1 \\
\hline $30 \mathrm{Zn}$ & 3 & 4 & 1 & 2 \\
\hline${ }_{31} \mathrm{Ga}$ & 1 & 4 & 3 & 2 \\
\hline${ }_{32} \mathrm{Ge}$ & 1 & 4 & 3 & 2 \\
\hline $33 \mathrm{As}$ & 1 & 4 & 2 & 3 \\
\hline${ }_{34} \mathrm{Se}$ & 1 & 4 & 2 & 3 \\
\hline${ }_{35} \mathrm{Br}$ & 1 & 4 & 2 & 3 \\
\hline${ }_{36} \mathrm{Kr}$ & 1 & 4 & 2 & 3 \\
\hline $37 \mathrm{Rb}$ & 1 & 4 & 2 & 3 \\
\hline${ }_{38} \mathrm{Sr}$ & 1 & 4 & 2 & 3 \\
\hline $39 \mathrm{Y}$ & 1 & 4 & 2 & 3 \\
\hline${ }_{40} \mathrm{Zr}$ & 3 & 2 & 1 & 4 \\
\hline${ }_{41} \mathrm{Nb}$ & 2 & 3 & 1 & 4 \\
\hline${ }_{42} \mathrm{Mo}$ & 2 & 3 & 1 & 4 \\
\hline${ }_{43} \mathrm{Tc}$ & 2 & 4 & 1 & 3 \\
\hline${ }_{44} \mathrm{Ru}$ & 3 & 2 & 1 & 4 \\
\hline${ }_{45} \mathrm{Rh}$ & 3.5 & 1 & 2 & 3.5 \\
\hline${ }_{46} \mathrm{Pd}$ & 3.5 & 2 & 1 & 3.5 \\
\hline${ }_{47} \mathrm{Ag}$ & 1.5 & 4 & 1.5 & 3 \\
\hline${ }_{48} \mathrm{Cd}$ & 3.5 & 2 & 1 & 3.5 \\
\hline${ }_{49} \mathrm{In}$ & 3 & 4 & 1.5 & 1.5 \\
\hline${ }_{50} \mathrm{Sn}$ & 3.5 & 3.5 & 2 & 1 \\
\hline${ }_{51} \mathrm{Sb}$ & 2 & 4 & 2 & 2 \\
\hline${ }_{52} \mathrm{Te}$ & 3 & 3 & 3 & 1 \\
\hline${ }_{53} \mathrm{I}$ & 3.5 & 1 & 3.5 & 2 \\
\hline${ }_{54} \mathrm{Xe}$ & 3 & 4 & 2 & 1 \\
\hline${ }_{55} \mathrm{Cs}$ & 2.5 & 4 & 2.5 & 1 \\
\hline${ }_{56} \mathrm{Ba}$ & 2 & 4 & 2 & 2 \\
\hline $\begin{array}{l}\text { Sum of } \\
\text { ranks: }\end{array}$ & 116 & 195 & 91 & 138 \\
\hline $\begin{array}{c}\text { Average } \\
\text { rank: }\end{array}$ & 2 & 4 & 1 & 3 \\
\hline
\end{tabular}


the cross sections for reaction products which cover very broad range of elements (from ${ }_{3} \mathrm{Li}$ to ${ }_{56} \mathrm{Ba}$ ) whereas the GEM2 gives comparably good predictions only for lithium and for the elements with large atomic number $(Z>40)$. This is illustrated by figs. $1-3$ which present the data and the theoretical cross sections for separate ranges of atomic number $Z$ of the products. Inspection of these figures allows to estimate qualitatively the agreement between the data and model predictions.

A very good quantitative reproduction of the experimental isotopic cross sections, i.e., such which results in the relative deviation between the data and the model cross sections smaller than $10 \%$, was achieved only for a small part of all the cross sections as it is shown in fig. 4 . The models ABLA07, SMM and GEMINI++ offer such a perfect reproduction of data for approximately $12 \%$ of the products whereas GEM2 only for approximately $4 \%$. This suggests that the models under consideration cannot be approved if the main criterion is perfect prediction of the cross sections for isotopically resolved reaction products.

Better but also not perfect prediction of the cross sections was found when the average agreement over isotopes of given element is considered. Such a quantitative comparison of the model and experimental cross sections was achieved by the application of a specific deviation factor (the $A$-factor) which was proposed in a previous paper [16] dealing with the reactions in the same nuclear system, i.e., ${ }^{136} \mathrm{Xe}+\mathrm{p}$ but at twice smaller energy (500 MeV per nucleon). The $Z$-dependence of the $A$-factor presented in fig. 5 shows that the production cross sections of elements with largest atomic numbers are equally well reproduced by all applied models whereas the situation changes for smaller atomic numbers. Then different models assure the best description for different ranges of the atomic number of products. The ranking of models based on achieved values of the $A$-factor was made (cf. table 1 ) which clearly shows that the averaged over isotopes and elements agreement between the data and experimental cross sections is the best for GEMINI++. The ABLA07 and SMM produced poorer average agreement and the GEM2 is the worst.

These conclusions agree with result obtained in the previous work [16] in which the analysis of the data measured at lower energy, i.e., $500 \mathrm{MeV}$ per nucleon for the same nuclear system were investigated. Then only elements with $Z>40$ were studied. The data were reproduced in the best way by GEMINI++, however the SMM model gave better description than ABLA07 whereas GEM2 also achieved the last position. The conclusion that the values of the $A$-factor are too big to claim that the studied models may be approved remains true also after the present analysis.

It is worth emphasizing that the ${ }^{136} \mathrm{Xe}+\mathrm{p}$ collisions at $1 \mathrm{GeV}$ per nucleon were studied intensively in the last years, both experimentally and theoretically [15,34-37]. In the first of these papers [15] the isotopic production cross sections measured at the GSI fragment separator were published for the full range of observed ejectiles, i.e., for elements from $Z=3(\mathrm{Li})$ to $Z=56(\mathrm{Ba})$. The second paper [34] was devoted to the analysis of the longitudinal velocity spectra of ejectiles from the above experiment with the aim to select isotopic cross sections for light ejectiles, i.e., elements from $Z=3(\mathrm{Li})$ to $Z=14(\mathrm{Si})$ on the basis of qualitatively different velocity spectra. The data from these publications, i.e., elemental cross sections and velocity spectra were compared by Mancusi et al. [35] with model calculations based on the INCL4.5 intranuclear cascade model coupled to the same deexcitation models as in the present work. It was found that no deexcitation model can reproduce the experimental longitudinal velocity distributions. On the other hand, the GEMINI++ model was found to be superior with respect to other deexcitation models like ABLA07, SMM and GEM2 in the description of the elemental production cross sections. This confirms the conclusions derived from the present investigation for more demanding isotopic cross sections.

The newer experiments performed with the SPALADIN setup at GSI $[36,37]$ allowed to identify in coincidence the projectile residue, the emitted fragments $(Z>2)$ and the average multiplicity of neutrons emitted in the de-excitation phase. Such exclusive observables should be much more restrictive to any of the spallation models than the elemental production cross sections analyzed by Mancusi et al. [35] or isotopic cross sections analyzed in the present work. The drawback of satisfactory description of the cross sections found in the present analysis shows a clear need for further improvements in theoretical models describing spallation reactions.

We thank Dr. D. Mancusi for providing us the latest version of the Liege intranuclear cascade code INCL ++ .

Open Access This is an open access article distributed under the terms of the Creative Commons Attribution License (http://creativecommons.org/licenses/by/4.0), which permits unrestricted use, distribution, and reproduction in any medium, provided the original work is properly cited.

\section{References}

1. R. Ramaty, R.E. Lingenfelter, Astrophys. Space Sci. 265, 71 (1999).

2. M. Salvatores, I. Slessarev, V. Berthou, Prog. Nucl. Energy 38, 167 (2001).

3. J.M. Carpenter, Nucl. Instrum. Methods 145, 91 (1977).

4. P. Napolitani, K.-H. Schmidt, A.S. Botvina, F. Rejmund, L. Tassan-Got, C. Villagrasa, Phys. Rev. C 70, 054607 (2004).

5. C. Villagrasa-Canton, A. Boudard, J.-E. Ducret, B. Fernandez, S. Leray, C. Volant, P. Armbruster, T. Enqvist, F. Hammache, K. Helariutta, B. Jurado, M.-V. Ricciardi, K.-H. Schmidt, K. Sümmerer, F. Vivés, O. Yordanov, L. Audouin, C.-O. Bacri, L. Ferrant, P. Napolitani, F. Rejmund, C. Stéphan, L. Tassan-Got, J. Benlliure, E. Casarejos, M. Fernandez-Ordoñez, J. Pereira, S. Czajkowski, D. Karamanis, M. Pravikoff, J.S. George, R.A. Mewaldt, N. Yanasak, M. Wiedenbeck, J.J. Connell, T. Faestermann, A. Heinz, A. Junghans, Phys. Rev. C 75, 044603 (2007). 
6. E. Le Gentil, T. Aumann, C.O. Bacri, J. Benlliure, S. Bianchin, M. Boöhmer, A. Boudard, J. Brzychczyk, E. Casarejos, M. Combet, L. Donadille, J.E. Ducret, M. Fernandez-Ordoñez, R. Gernhäuser, H. Johansson, K. Kezzar, T. Kurtukian-Nieto, A. Lafriakh, F. Lavaud, A. Le Févre, S. Leray, J. Lühning, J. Łukasik, U. Lynen, W.F.J. Müller, P. Pawłowski, S. Pietri, F. Rejmund, C. Schwarz, C. Sfienti, H. Simon, W. Trautmann, C. Volant, O. Yordanov, Phys. Rev. Lett. 100, 022701 (2008).

7. F. Rejmund, B. Mustapha, P. Armbruster, J. Benlliure, M. Bernas, A. Boudard, J.P. Dufour, T. Enqvist, R. Legrain, S. Leray, K.-H. Schmidt, C. Stéphan, J. Taieb, L. TassanGot, C. Volant, Nucl. Phys. A 683, 540 (2001).

8. J. Benlliure, P. Armbruster, M. Bernas, A. Boudard, J.P. Dufour, T. Enqvist, R. Legrain, S. Leray, B. Mustapha, F. Rejmund, K.-H. Schmidt, C. Stéphan, L. Tassan-Got, C. Volant, Nucl. Phys. A 683, 513 (2001).

9. J. Benlliure, P. Armbruster, M. Bernas, A. Boudard, T. Enqvist, R. Legrain, S. Leray, F. Rejmund, K.-H. Schmidt, C. Stéphan, L. Tassan-Got, C. Volant, Nucl. Phys. A 700, 469 (2002).

10. W. Wlazło, T. Enqvist, P. Armbruster, J. Benlliure, M. Bernas, A. Boudard, S. Czájkowski, R. Legrain, S. Leray, B. Mustapha, M. Pravikoff, F. Rejmund, K.-H. Schmidt, C. Stéphan, J. Taieb, L. Tassan-Got, C. Volant, Phys. Rev. Lett. 84, 5376 (2000).

11. L. Audouin, L. Tassan-Got, P. Armbruster, J. Benlliure, M. Bernas, A. Boudard, E. Casarejos, S. Czajkowski, T. Enqvist, B. Fernandez-Dominguez, B. Jurado, R. Legrain, S. Leray, B. Mustapha, J. Pereira, M. Pravikoff, F. Rejmund, M.-V. Ricciardi, K.-H. Schmidt, C. Stéphan, J. Taieb, C. Volant, W. Wlazło, Nucl. Phys. A 768, 1 (2006).

12. M. Bernas, P. Armbruster, J. Benlliure, A. Boudard, E. Casarejos, S. Czajkowski, T. Enqvist, R. Legrain, S. Leray, B. Mustapha, P. Napolitani, J. Pereira, F. Rejmund, M.-V. Ricciardi, K.-H. Schmidt, C. Stéphan, J. Taieb, L. TassanGot, C. Volant, Nucl. Phys. A 725, 213 (2003).

13. M. Bernas, P. Armbruster, J. Benlliure, A. Boudard, E. Casarejos, T. Enqvist, A. Kelic, R. Legrain, S. Leray, J. Pereira, F. Rejmund, M.-V. Ricciardi, K.-H. Schmidt, C. Stéphan, J. Taieb, L. Tassan-Got, C. Volant, Nucl. Phys. A 765, 197 (2006).

14. M.V. Ricciardi, P. Armbruster, J. Benlliure, M. Bernas, A. Boudard, S. Czajkowski, T. Enqvist, A. Kelic, S. Leray, R. Legrain, B. Mustapha, J. Pereira, F. Rejmund, K.-H. Schmidt, C. Stephan, L. Tassan-Got, C. Volant, O. Yordanov, Phys. Rev. C 73, 014607 (2006).

15. P. Napolitani, K.-H. Schmidt, L. Tassan-Got, P. Armbruster, T. Enqvist, A. Heinz, V. Henzl, D. Henzlova, A. Kelić, R. Pleskač, M.V. Ricciardi, C. Schmitt, O. Yordanov, L. Audouin, M. Bernas, A. Lafriaskh, F. Rejmund, C. Stéphan, J. Benlliure, E. Casarejos, M. Fernandez Ordonez, J. Pereira, A. Boudard, B. Fernandez, S. Leray, C. Villagrasa, C. Volant, Phys. Rev C 76, 064609 (2007).

16. S.K. Sharma, B. Kamys, F. Goldenbaum, D. Filges, Eur. Phys. J. A 53, 150 (2017).
17. L. Giot, J.A. Alcántara-Núñez, J. Benlliure, D. PérezLoureiro, L. Audouin, A. Boudard, E. Casarejos, T. Enqvist, J.E. Ducret, B. Fernández-Domínguez, M. Fernández Ordóñez, F. Farget, A. Heinz, V. Henzl, D. Henzlova, A. Kelić-Heil, A. Lafriaskh, S. Leray, P. Napolitani, C. Paradela, J. Pereira, M.V. Ricciardi, C. Stéphan, K.H. Schmidt, C. Schmitt, L. Tassan-Got, C. Villagrasa, C. Volant, O. Yordanov, Nucl. Phys. A 899, 116 (2013).

18. D. Mancusi, A. Boudard, J. Cugnon, J.-C. David, P. Kaitaniemi, S. Leray, Phys. Rev. C 90, 054602 (2014).

19. A. Kelić, M.V. Ricciardi, K.-H. Schmidt, in Proceedings of the Joint ICTP-IAEA Advanced Workshop on Model Codes for Spallation Reactions, ICTP Trieste, Italy, 4-8 February 2008, edited by D. Filges et al., IAEA INDC(NDS)-530, (IAEA, Vienna, 2008) p. 181, http:// www-nds . iaea.org/reports-new/indc-reports/indcnds/indc-nds-0530.pdf, arXiv:0906.4193[nucl-th].

20. S. Furihata, Nucl. Instrum. Methods B 171, 251 (2000).

21. S. Furihata, T. Nakamura, J. Nucl. Sci. Technol. Suppl. 2, 758 (2002).

22. R.J. Charity et al., Nucl. Phys. A 483, 371 (1988).

23. R.J. Charity, Phys. Rev. C 82, 014610 (2010).

24. A.S. Botvina, A.S. Iljinov, I.N. Mishustin, Yad. Fiz. 42, 1127 (1985) Sov. J. Nucl. Phys. 42, 712 (1985).

25. A.S. Botvina, A.S. Iljinov, I.N. Mishustin, J.P. Bondorf, R. Donangelo, K. Sneppen, Nucl. Phys. A 475, 633 (1987).

26. A.S. Botvina, A.S. Iljinov, I.N. Mishustin, Nucl. Phys. A 507, 649 (1990).

27. J.P. Bondorf, A.S. Botvina, A.S. Iljinov, I.N. Mishustin, K. Sneppen, Phys. Rep. 257, 133 (1995).

28. A. Boudard, J. Cugnon, J.-C. David, S. Leray, D. Mancusi, Phys. Rev. C 87, 014606 (2013).

29. V.F. Weisskopf, D.H. Ewing, Phys. Rev. 57, 472 (1940).

30. F. Atchison, in Proceedings of the Meeting on Targets for Neutron Beam Spallation Source, edited by G. Bauer, Jülconf-34 (KFA Jülich Germany, 1980), p. 17.

31. W. Hauser, H. Feshbach, Phys. Rev. 87, 366 (1952).

32. L.G. Moretto, G.J. Wozniak, Prog. Part. Nucl. Phys. 21, 401 (1988).

33. N. Bohr, J.A. Wheeler, Phys. Rev. 56, 426 (1939).

34. P. Napolitani, K.-H. Schmidt, L. Tassan-Got, J. Phys. G 38, 115006 (2011).

35. D. Mancusi, A. Boudard, J. Cugnon, J.-Ch. David, T. Gorbinet, S. Leray, Phys. Rev. C 84, 064615 (2011).

36. T. Gorbinet, T. Aumann, S. Bianchin, O. Borodina, A. Boudard, C. Caesar, E. Casajeros, B. Czech, J.-É. Ducret, S. Hlavac, N. Kurz, C. Langer, T. Le Bleis, S. Leray, J. Łukasik, P. Pawłowski, S. Pietri, M.-D. Salsac, H. Simon, M. Veselsky, Y. Ayyad, O. Yordanov, Phys. Scr. T150, 014015 (2012).

37. O. Yordanov, J.-É. Ducret, T. Gorbinet, S. Leray, A. Boudard, M.D. Salsac, J. Phys.: Conf. Ser. 366, 012051 (2012). 